\title{
A budget impact analysis of the VENTANA PD-L1 SP142 immunohistochemistry assay versus the Dako PD-L1 IHC 22C3 assay in patients with advanced or metastatic triple-negative breast cancer treated with atezolizumab in combination with nab-paclitaxel
}

Cristian Scatena ${ }^{1}$, Roberto Ravasio ${ }^{2}$, Paola Raimondo ${ }^{3}$, Mario Giuliano ${ }^{4}$

Division of Pathology, Department of Translational Research and New Technologies in Medicine and Surgery, University of Pisa, Pisa, Italy

HEOR \& RWE Lead, Market Access Provider, Milano, Italy

Market Access Analyst, Market Access Provider, Milano, Italy

${ }^{4}$ Department of Clinical Medicine and Surgery, University of Naples "Federico II", Napoli, Italy

\begin{abstract}
OBJECTIVE: To estimate the budget impact determined by the adoption of two different diagnostic strategies, SP142 assay or 22C3 assay, in the identification (in terms of PD-L1 status) of patients with $\mathrm{mTNBC}$ eligible for treatment with atezolizumab in combination with nab-paclitaxel.

METHODS: The budget impact analysis (BIA) was conducted using a budget impact model (BIM) considering the Italian National Health Service's (iNHS) perspective. The analysis assessed only the direct medical cost (tissue biopsy, PD-L1 assay, specialist visit, pharmacological treatment with atezolizumab in combination with nab-paclitaxel) of patients with PDL1 positive mTNBC, and management of the adverse events associated with the pharmacological treatment administered. The BIM also considered the clinical benefits (progression free survival, PFS) resulting from the drug therapy administered on the basis of the results of the post-hoc analysis of the IMpassion 130 clinical trial. The BIA was conducted over a 1-year time horizon. The median cost per patient in the progression-free state was also calculated. The costs were calculated using the net ex-factory prices (cancer drugs) and regional or national tariffs (tissue biopsy, PD-L1 assay, specialist visit and adverse events management). A sensitivity analysis was conducted to evaluate the base case result.

RESULTS: The SP142 assay diagnostic pathway would result in a reduction of the iNHS expenditure of approximately 5.6 million euros $(-12 \%)$. Almost all of the reduction in iNHS expenditure would be determined by the lower number of patients treated (SP142: 689 patients vs 22C3: 786 patients) with immunotherapy $(-€ 5,530,871)$. Compared with 22C3 assay, the SP142 assay shows a cost per PFS month reduction of $€ 736$ ( $€ 7,010$ vs $€ 7,746)$.

CONCLUSIONS: The use of the SP142 assay proved to be cost-effective compared to the $22 \mathrm{C} 3$ assay; the SP142 assay can support the choice of the most appropriate cancer drug, maximizing the effectiveness and minimizing the waste of healthcare resources.
\end{abstract}

\section{Keywords}

VENTANA PD-L1 SP142 assay, Triple-negative breast cancer, Atezolizumab, Budget impact, Italian NHS

\section{INTRODUCTION}

Breast cancer (BC) is a heterogeneous disease, mainly characterized by three subtypes defined by the estrogen receptor (ER) and progesterone (PR) immunohistochemical expression and by the amplification - or overexpression - of the human epidermal growth factor receptor 2 (HER2). These subtypes are hormone receptor positive (HR+)/HER2-negative, HER2-positive and triple negative [TN]) [1].

Triple-negative breast cancer (TNBC) does not express ER, PR and HER2 [2]. TNBC accounts for $10-15 \%$ of all breast cancers and, among the different subtypes, has the worst

Corresponding author Roberto Ravasio ravasio@maprovider.com Received: 20 May 2021 Accepted: 13 July 2021 Published: 26 July 2021 
prognosis [1,3] due to a high risk of relapse [4] and a short overall survival (OS) of patients already in the metastatic phase, whose median value is around 13 months [5].

For years, the reference therapy for patients with unresectable locally advanced or metastatic TNBC (mTNBC) has been systemic chemotherapy $[1,3]$. The need to improve the therapeutic outcomes - and, at the same time, the advances made in the molecular characterization of mTNBC - drove the scientific research to develop new drugs. Due mainly to the successes of immune checkpoint inhibitors (immunotherapies) in other tumors, PD-L1 and PD-1 inhibitors have been investigated as potential treatments for mTNBC [3]. The overexpression of PD-L1 detected in patients with mTNBC, compared to other subtypes of breast cancer, has in fact promoted the development of these immunotherapies, capable of blocking the immune checkpoint involved, that is the interaction between PD-L1 and its PD-1 receptor [3]. Programmed death ligand-1 (PD-L1) is a transmembrane protein present on the surface of both immune and tumor cells by interacting with its two programmed death receptors PD-1 and B7.1, located on the surface of T cells, it downregulates the antitumor immune response $[1,6]$. Immunological drugs, such as atezolizumab, are able to block this molecular mechanism by which the tumor resists the attack of the immune system [6]. Atezolizumab is an engineered humanized monoclonal antibody which, by binding to PD-L1, prevents its interaction with the PD-1 receptor, unblocking the inhibition of the immune response mediated by PD-L1/PD-1 and reactivating the antitumor immune response [7]. Atezolizumab, in combination with the chemotherapy agent nab-paclitaxel, has been approved by EMA - and subsequently reimbursed by AIFA (Official Gazette [GU] of July 28, 2020) - for the treatment of adult patients with unresectable locally advanced or metastatic TNBC whose tumors have a PD-L1 expression $\geq 1 \%$ and who have not received any prior chemotherapy for metastatic disease [8].

In the era of precision medicine, it becomes essential to identify predictive factors, in order to select the patients who can benefit from the immunological treatment, evaluating not only the clinical aspect, but also the cost associated with these molecules, as well as their impact on healthcare systems. For this reason, before administering immunotherapy, patients with mTNBC should undergo a diagnostic assay to assess the expression (or not) of PD-L1, in order to identify their eligibility for treatment. There are various assays for the determination of PD-L1 expression but, at the moment, the only one authorized for the indication for the use of atezolizumab in mTNBC is the VENTANA PD-L1 SP142 immunohistochemistry (IHC) assay (breviter SP142 assay), an immunohistochemical assay which uses an anti-PDL1 rabbit monoclonal primary antibody for the recognition of the PD-L1 protein [9]. SP142 evaluates the portion of the tumor area occupied by tumor-infiltrating immune cells (IC) with PD-L1 expression (\% IC); positivity for the assay is determined by the presence of a perceptible PD-L1 staining of any intensity in the ICs on a percentage $\geq 1$ of the tumor area occupied by tumor cells and the associated intratumoral and contiguous peritumoral stroma [9].

The performance of the SP142 assay was examined in IMpassion130, a phase III, doubleblind, two-arm, multicenter, international, randomized, placebo-controlled study conducted to evaluate the efficacy and safety of atezolizumab in combination with nab-paclitaxel in patients with unresectable locally advanced or metastatic TNBC who had not received prior chemotherapy for metastatic disease [3]. A recent post-hoc analysis of the IMpassion130 study evaluated in the biomarker-evaluable subpopulation (population suitable for the predictive assay for PD-L1) the concordance and association with the clinical efficacy of atezolizumab plus nab-paclitaxel of the SP142 assay and that of the Dako PD-L1 IHC 22C3 pharmDx assay (breviter 22C3 assay), currently used as a diagnostic assay for other immunological treatments $[10,11]$. Positivity for the $22 \mathrm{C} 3$ assay was defined by a combined positive score (CPS) $\geq 10[10,11]$. As part of the IMpassion130 study population, the biomarker-evaluable subpopulation was treated with nab-paclitaxel combined with either atezolizumab or placebo, in order to evaluate progression-free survival (PFS) [10,11].

\section{OBJECTIVE}

The purpose of this analysis was to estimate the budget impact on the Italian National Health Service (iNHS) determined by the adoption of two different diagnostic strategies, SP142 assay or 22C3 assay, in the identification (in terms of PD-L1 status) of patients with $\mathrm{mTNBC}$ eligible for treatment with atezolizumab in combination with nab-paclitaxel.

\section{MATERIALS AND METHODS}

The budget impact analysis (BIA) was conducted using a budget impact model (BIM) considering the iNHS's perspective. The analysis assessed only the direct medical costs such 
as: tissue biopsy, PD-L1 assay, specialist visit, pharmacological treatment (atezolizumab in combination with nab-paclitaxel) of patients with PD-L1 positive mTNBC, and management of the adverse events associated with the pharmacological treatment administered. The BIM also considered the clinical benefits, expressed in terms of PFS, resulting from the drug therapy administered on the basis of the results of the post-hoc analysis of the IMpassion 130 clinical trial $[10,11]$. The BIA was conducted over a 1-year time horizon, assuming that, given the poor prognosis of the metastatic disease, for each patient with mTNBC the permanence in the state of PFS ended within this period. This hypothesis probably represents a limit compared to what happens in clinical practice, where patients could be characterized by a different PFS; however, it allows to estimate the potential economic impact of the two diagnostic strategies with respect to a defined time frame which, in the decisionmaking processes, is critically important. The analysis was carried out following the ISPOR (International Society for Pharmacoeconomics and Outcomes Research) good practice for budget impact analysis $[12,13]$.

Based on the results of the BIA, the median cost per patient in the progression-free state was also calculated. This cost was first calculated by dividing - for each of the two strategies - the total cost of treatment (tissue biopsy, PD-L1 assay, specialist visit, immunotherapy, administration of immunotherapy and management of adverse events) by the number of patients positive to the PD-L1 assay, then dividing this cost of treatment by the median number of months in PFS, obtained from the post-hoc analysis of the IMpassion130 clinical study $[10,11]$.

\section{Model input data}

\section{Population}

Table I describes the estimated number of patients considered in the BIA. It was calculated starting from the number of Italian residents in January 2020 [14]. The incidence of new diagnoses of $\mathrm{BC}$ is $0.1 \%$ [15], of which $12.5 \%$ are triple negative [16]. Five percent of the new TNBC diagnoses present a de novo locally advanced or metastatic stage of disease [17], while $25 \%$ have a locally advanced or metastatic stage of disease following a recurrence [18]. Finally, it is assumed that $72 \%$ of potentially eligible patients undergo the PD-L1 assay [19]. The latter percentage was estimated considering the portion of patients who, due to clinical characteristics, are not eligible for immunotherapy (e.g., particular comorbidities) or for testing (e.g., lack of tissue) [19]. Therefore, the BIM considered 1,485 patients.

\section{Model structure}

Figure 1 shows the structure of the BIM. Scenario 1 reflects the expenditure associated with the identification of PD-L1 expression with the SP142 assay and with the subse-

\begin{tabular}{|c|c|c|c|}
\hline & Parameters & Year 1 & Source \\
\hline A & Population (1 January 2020) (n) & $59,641,488$ & {$[14]$} \\
\hline B & Incidence BC (\%) & $0.1 \%$ & [15] \\
\hline C & Patients BC (n) & 54,976 & $C=A \times B$ \\
\hline $\mathrm{D}$ & Incidence TNBC (\%) & $12.5 \%$ & [16] \\
\hline E & Patients TNBC (n) & 6,872 & $E=D \times C$ \\
\hline $\mathrm{F}$ & Incidence mTNBC de novo (\%) & $5.0 \%$ & [17] \\
\hline G & Patients mTNBC de novo (n) & 344 & $G=E \times F$ \\
\hline $\mathrm{H}$ & $\begin{array}{l}\text { Incidence mTNBC following } \\
\text { recurrence (\%) }\end{array}$ & $25.0 \%$ & [18] \\
\hline I & $\begin{array}{l}\text { Patients } m T N B C \text { following } \\
\text { recurrence }(n)\end{array}$ & 1,718 & $\mathrm{I}=\mathrm{E} \times \mathrm{H}$ \\
\hline L & $\begin{array}{l}\text { Patients potentially eligible for the } \\
\text { PD-L1 assay (n) }\end{array}$ & 2,062 & $L=G+I$ \\
\hline M & $\begin{array}{l}\text { Patients actually eligible for the } \\
\text { PD-L1 assay (\%) }\end{array}$ & $72.0 \%$ & [19] \\
\hline N & $\begin{array}{l}\text { Patients actually eligible for the } \\
\text { PD-L1 assay (n) }\end{array}$ & 1,485 & $N=L \times M$ \\
\hline
\end{tabular}

Table I. Estimated population of the budget impact model

$\mathrm{BC}=$ breast cancer; TNBC = triple-negative breast cancer; $\mathrm{mTNBC}=$ unresectable locally advanced or metastatic TNBC

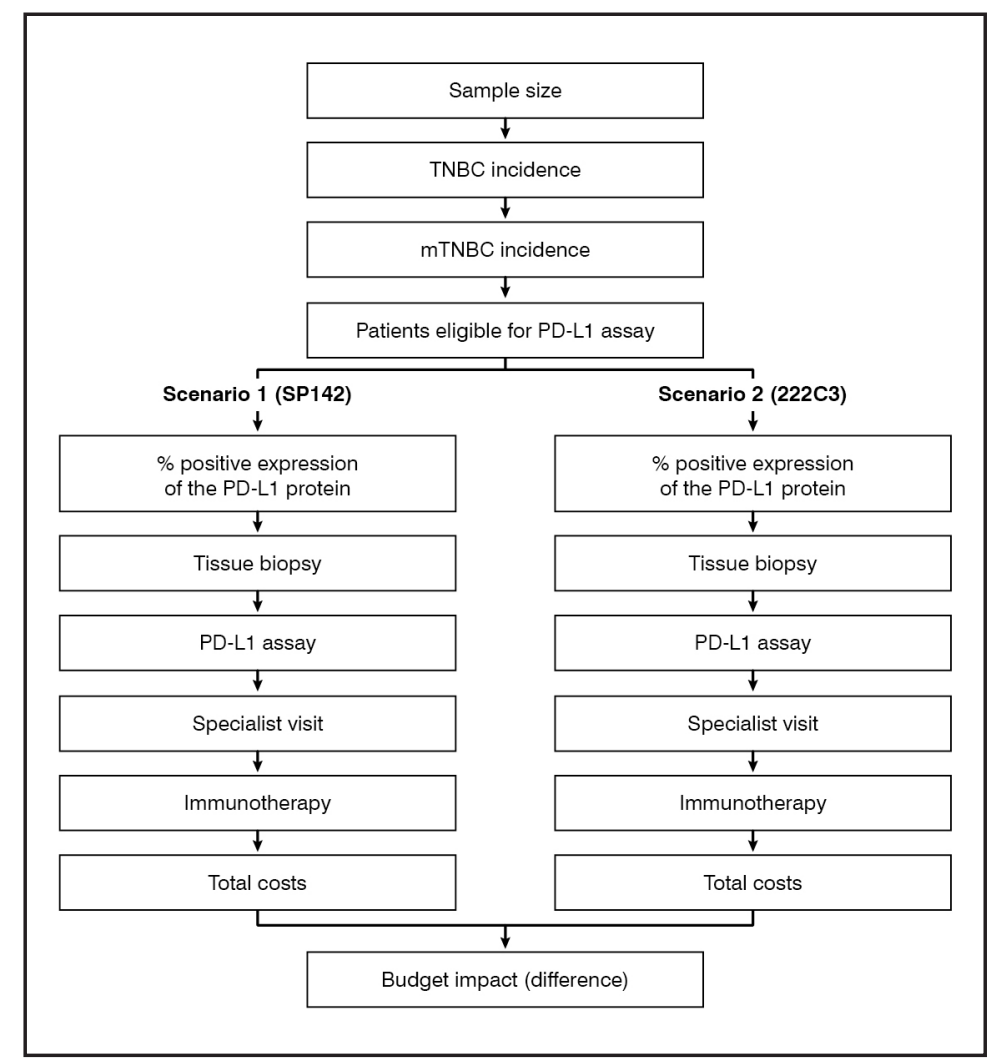

Figure 1. Model structure: SP142 vs 22C3

$\mathrm{TNBC}=$ triple-negative breast cancer; $\mathrm{mTNBC}=$ unresectable locally advanced or metastatic TNBC 


\begin{tabular}{lccc}
\hline \multicolumn{1}{c}{ Parameters } & BEP, $\mathbf{n}(\%)$ & $\begin{array}{c}\text { Atezolizumab + nab-paclitaxel, } \\
\text { median PFS (months) }\end{array}$ & Source \\
\hline SP142 & & & \\
IC $\geq 1 \%$ & $285(46.4)$ & 8.3 & {$[10,11]$} \\
$I C<1 \%$ & $329(53.6)$ & 5.7 & {$[10,11]$} \\
$\mathbf{2 2 C 3}$ & & & {$[10,11]$} \\
CPS $\geq 10$ & $325(52.9)$ & 7.5 & {$[10,11]$} \\
CPS $<10$ & $289(47.1)$ & 5.8 & \\
\hline
\end{tabular}

Table II. Clinical data PD-L1 assay evaluated within IMpassion 130 study population

$\mathrm{BEP}=$ biomarker-evaluable population; $\mathrm{IC}=$ immune cells; $\mathrm{CPS}=$ combined positive score; PFS = progression free survival

quent treatment with atezolizumab in combination with nab-paclitaxel of patients diagnosed with $\mathrm{mTNBC}$ and eligible for therapy. Scenario 2 reflects instead the expenditure associated with the identification of PD-L1 expression with the 22C3 assay and with the subsequent treatment with atezolizumab in combination with nab-paclitaxel of patients diagnosed with mTNBC and eligible for therapy. In both scenarios, the eligible mTNBC patient undergoes the PD-L1 assay (SP142 or 22C3) after a tissue biopsy. Subsequently - assuming the positive expression of the PD-L1 protein, and after a specialist visit - the patient is treated with atezolizumab in combination with nab-paclitaxel.

\section{Clinical data}

The clinical data used for the comparison between the SP142 and the $22 \mathrm{C} 3$ assay were extrapolated from the post-hoc analysis of the clinical study IMpassion130 [10,11]. The post$h o c$ analysis considered the population suitable (Biomarker-Evaluable Population, BEP) for the PD-L1 predictive assay (SP142 or 22C3). With reference to a sample of 614 patients, $46.4 \%(\mathrm{n}=285)$ and $52.9 \%(\mathrm{n}=325)$ tested positive to the SP142 assay and for the $22 \mathrm{C} 3$ assay, respectively, where positivity for PD-L1 status was defined by CI $\geq 1 \%$ for the SP142 assay and by CPS $\geq 10$ for the $22 \mathrm{C} 3$ assay (Table II). The $22 \mathrm{C} 3$ assay identified a larger positive PD-L1 population than that identified by the SP142 assay, of which the latter is a subgroup (positive percentage agreement 98\%) [10,11]. Patients 22C3 PD-L1 positive (CPS $\geq 10$ ) do not achieve the same benefit (PFS) with atezolizumab in combination with nab-paclitaxel as those SP142 PD-L1 positive (CI $\geq 1 \%$ ) [10,11]; the post-hoc analysis of the IMpassion130 clinical trial reports a median PFS of 8.3 months for SP142 positive patients and 7.5 months for those positive to the $22 \mathrm{C} 3$ assay (Table II) $[10,11]$.

\section{Healthcare consumption and costs}

Table III shows the healthcare consumption and the related unit costs used in the BIM to evaluate costs associated with the diagnostic pathway including the tissue biopsy, the PD-L1 assay (SP142 or 22C3) and the specialist visit. The tissue biopsy cost was evaluated considering as a proxy the tariff 91.42.B "histopathological examination of the breast-excision of neoformation" reimbursed by Tuscany Region [20]. The PD-L1 assay cost was assessed considering as a proxy the tariff reimbursed by Piedmont Region for the execution of the panel of prognostic and predictive factors of response to the therapy for breast malignant tumors (code 91.47.6) [21]. The cost of a specialist visit, instead, was calculated by applying as a proxy the national tariff reimbursed for the general visit (code 89.7) [22].

Table IV reports the administration scheme for atezolizumab in combination with nab-paclitaxel; this scheme reflects the indications of the IMpassion 130 clinical study [3]. The costs of related consumptions were calculated using the ex-factory prices, net of the temporary discounts required by law (AIFA Resolution of July 3, 2006, Official Gazette (GU) no. 156 of July 7, 2006, and subsequent AIFA Resolution of February 9, 2007, Official Gazette (GU) no. 57 of March 9, 2007 and extensions), equal to $€ 3,221.93$ for an $840 \mathrm{mg}$ vial of atezolizumab and to $€ 221.11$ for a $100 \mathrm{mg}$ vial of nab-paclitaxel [8].

The cost of drug intravenous administration was assessed considering the MAC01 tariff (package rates for complex and high-resource-integration outpatient macro-activity - MAC) reimbursed by Lombardy Region, equal to $€ 44.00$ [23].

Following what reported in the IMpassion130 study [3], Table $\mathrm{V}$ shows the frequency of grade $\geq 3$ adverse events (fatigue, peripheral neuropathy and neutropenia) associated with the administration of atezolizumab in combination with nab-paclitaxel, and the related unit cost. 
When available, cost of adverse events came from national literature [24,25] or, alternatively, referring to the DRG tariffs, when the management of grade $\geq 3$ adverse event required hospitalization [26].

\section{Model output data}

\section{Budget Impact}

Based on the eligible population and the estimated costs for the diagnostic pathway (tissue biopsy, PD-L1 assay and specialist visit) and for pharmacological treatment (drug, administration and adverse events), the BIA provides for the two strategies, over 1-year time horizon, the number of eligible patients, the number of PD-L1 cases correctly identified and the total cost of treatment. In addition, considering the associated clinical benefit, the difference in iNHS expenditure generated by the two different diagnostic strategies is presented also in terms of median cost per month in PFS.

\section{Sensitivity analysis}

The sensitivity analysis was conducted to assess the degree of uncertainty of the base case results [27]. A One-Way Sensitivity Analysis (OWSA) was conducted to the following parameters. The Confidence Interval/range or, alternatively, a variation of $\pm 10 \%$ for incidence data or a variation of $\pm 5 \%$ for assay was used to run the OWSA:

- annual incidence of TNBC (10-15\%);

- annual incidence of de novo $\mathrm{mTNBC}( \pm 10 \%)$;

- annual incidence of mTNBC following recurrence $( \pm 10 \%)$;

- incidence of mTNBC patients actually eligible for the assay $( \pm 10 \%)$;

- percentage of patients positive to the PD-L1 SP142 assay $( \pm 5 \%)$;

- percentage of patients positive to the PD-L1 $22 \mathrm{C} 3$ assay $( \pm 5 \%)$.

Since the net ex-factory price may not reflect the actual selling price to the iNHS, a scenario analysis was conducted to assess the impact that different percentages of discount on the selling price of atezolizumab to the iNHS would have on the overall treatment cost. Three alternative scenarios were considered in which a $5 \%, 10 \%$ or $15 \%$ discount was applied to the base case price of atezolizumab.

Since it was not possible to distinguish between neutropenia and febrile neutropenia with reference to the data from Impassion 130 , and since grade $\geq 3$ non-febrile neutropenia may not involve any treatment or hospitalization, in the sensitivity analysis a scenario was assumed which excluded the costs associated with the management of neutropenia.

\section{RESULTS}

\section{Budget impact}

Due to a greater specificity, the diagnostic pathway that takes into account SP142 assay would result in a reduction of the iNHS expenditure of approximately 5.6 million euros $(-12 \%)$ (Table VI). Almost all of the reduction in iNHS expenditure would be determined by the lower number of patients treated (SP142: 689 patients vs 22C3: 786 patients) with immunotherapy $(€-5,530,871)$. The lower number of patients treated with immunotherapy would also lead to a reduction, albeit less significant $(€-39,066)$, in the iNHS expenditure for the management of the related adverse events. Conversely, the expenditure for the diagnostic path management (tissue biopsy, PD-L1 assay and specialist visit) would not determine any incremental cost between the two strategies considered. 


\begin{tabular}{lcccc}
\hline \multirow{2}{*}{ Parameters } & SP142 & 22C3 & \multicolumn{2}{c}{ Difference SP142 vs 22C3 } \\
\cline { 4 - 5 } & & 495,990 & Absolute & \% \\
\hline Tissue biopsy + PD-L1 assay $(€)$ & 495,990 & 30,680 & 0 & 0 \\
Specialist visit $(€)$ & 30,680 & $44,817,158$ & $-5,530,871$ & -12 \\
Treatment $(€)$ & $39,286,288$ & 316,553 & $-39,066$ & -12 \\
Adverse events $(€)$ & 277,486 & $\mathbf{4 5 , 6 6 0 , 3 8 2}$ & $\mathbf{- 5 , 5 6 9 , 9 3 6}$ & $\mathbf{- 1 2}$ \\
Total costs $(\boldsymbol{\epsilon})$ & $\mathbf{4 0 , 0 9 0 , 4 4 5}$ & &
\end{tabular}

Table VI. BIM results

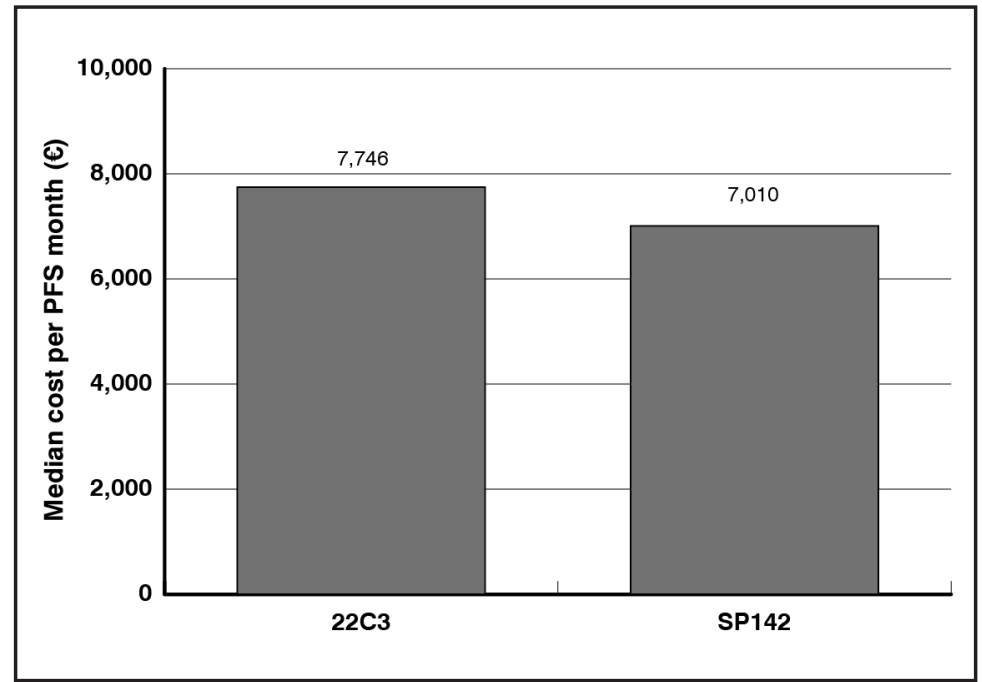

Figure 2. Median cost per month in PFS

\begin{tabular}{lccc}
\hline \multirow{1}{*}{ Parameter } & Variation & \multicolumn{2}{c}{ Budget impact (€) } \\
\cline { 3 - 4 } & & $\begin{array}{c}\text { Lower } \\
\text { extreme }\end{array}$ & $\begin{array}{c}\text { Upper } \\
\text { extreme }\end{array}$ \\
\hline Annual incidence TNBC & $(10 \%-15 \%)$ & $-4,478,918$ & $-6,660,955$ \\
$\begin{array}{l}\text { Annual incidence de novo } \\
\text { TNBC }\end{array}$ & $\pm 10 \%$ & $-5,455,092$ & $-5,684,780$ \\
$\begin{array}{l}\text { Annual incidence TNBC } \\
\text { following recurrence }\end{array}$ & $\pm 10 \%$ & $-5,053,138$ & $-6,029,313$ \\
$\begin{array}{l}\text { Incidence of mTNBC patients } \\
\text { actually eligible for the assay }\end{array}$ & $\pm 10 \%$ & $-4,995,716$ & $-6,086,735$ \\
$\begin{array}{l}\text { Percentage of patients positive } \\
\text { to the PD-L1 SP142 assay }\end{array}$ & $\pm 5 \%$ & $-7,522,285$ & $-3,560,166$ \\
$\begin{array}{l}\text { Percentage of patients positive } \\
\text { to the PD-L1 22C3 assay }\end{array}$ & $\pm 5 \%$ & $-3,330,477$ & $-7,809,395$ \\
$\begin{array}{l}\text { Exclusion of the cost of } \\
\text { neutropenia }\end{array}$ & & \multicolumn{2}{c}{$-5,537,711$} \\
\hline \begin{tabular}{l} 
Base case \\
\hline
\end{tabular}
\end{tabular}

Table VII. Sensitivity analysis (OWSA)

TNBC = triple-negative breast cancer; $\mathrm{mTNBC}=$ unresectable locally advanced or metastatic TNBC
Figure 2 reports the median cost per month in PFS. The patient with SP142 assay shows a cost per PFS month reduction of $€$ 736 ( $€ 7,010$ vs $€ 7,746)$.

\section{Sensitivity analysis}

The OWSA results show that the proportion of patients positive to the PD-L1 assay (SP142 or 22C3) constitutes the main driver of the BIA (Table VII).

Considering a $5 \%$ discount on the selling price of atezolizumab, the difference of the iNHS expenditure between the two diagnostic strategies would be reduced, compared to the base case, by $€ 237,651$ ( $€-5,332,286)$. Considering a $10 \%$ or $15 \%$ reduction in the selling price, there would be - compared to the base case - a decrease in the difference of the iNHS expenditure between the two strategies equal to $€ 475,302$ ( $€-5,094,635)$ and $€$ $712,952$ ( $€-4,856,984)$, respectively.

The exclusion of grade $\geq 3$ neutropenia cost would result in a slight reduction in the savings associated with SP142 assay compared to the base case (sensitivity analysis: $€$ $-5,537,711$; base case: $€-5,569,936)$.

\section{DISCUSSION}

A BIM was developed to evaluate the economic impact on the iNHS determined by the adoption of two different diagnostic strategies (SP142 assay or 22C3 assay) for the identification of PD-L1 overexpression in patients with $\mathrm{mTNBC}$. The BIM estimated the clinical and economic consequences in terms of iNHS expenditure and median cost per PFS month.

The analysis highlighted the clinical and economic advantage of using the SP142 assay over the 22C 3 assay as a complementary diagnostic for the administration of atezolizumab in combination with nab-paclitaxel to mTNBC patients. The diagnostic pathway involving the administration of the SP142 assay would result in a $€ 5,569,936$ reduction in the iNHS expenditure compared to the adoption of the $22 \mathrm{C} 3$ assay ( $€ 40,090,445$ vs $€ 45,660,382)$. Such result would be attributable to the lower number of patients defined as eligible for immunotherapy treatment (698 vs 786) based on the greater specificity of the SP142 assay (46.4\%) in identifying PD-L1 overexpression in mTNBC patients compared to the $22 \mathrm{C} 3$ assay (52.9\%). In addition to containing pharmaceutical 
expenditure, the SP142 assay correctly identifies the patients who are most likely to benefit from immunotherapy improving the iNHS efficiency. In fact, the cost per PFS month would be lower in SP142 assay patients compared to those with $22 \mathrm{C} 3$ assay $(€ 7,010$ vs $€ 7,746)$. The use of a more specific diagnostic assay would make immunotherapy more efficient, thus avoiding it being wasted on patients who would not benefit from it.

As often happens when a simulation model is used to conduct a comparison between alternative choices, it is appropriate to interpret the results in view of some observations. The epidemiological analysis - used to identify the mTNBC patients eligible for the PD-L1 assay - was built using data from different literature sources and, in their absence, by making some assumptions based on market research. Since it is not possible to verify the effective representativeness of our patient flow with other national experiences, the sensitivity analysis was conducted to check our epidemiological assumptions. No overestimation or underestimation of the epidemiological parameters would seem to have such an impact as to make the result of the base case vary by more than $\pm 20 \%$.

The IMpassion 130 post-hoc analysis data highlighted a greater specificity of the SP142 assay $[10,11]$. In the absence of any other available clinical evidence, the sensitivity analysis evaluated the impact deriving from the variation in the percentage of patients positive for PD-L1. A 5\% reduction or increase in the number of PD-L1 positive patients with the SP142 assay would result in a $35 \%$ reduction or increase in the base case result, respectively. A $5 \%$ reduction or increase in the number of PD-L1 positive patients with the $22 \mathrm{C} 3$ assay would result in a $40 \%$ reduction or increase in the base case result, respectively.

Although the net ex-factory price may not reflect the actual iNHS selling price of atezolizumab, a scenario analysis was conducted to assess the impact that different discount percentages $(5 \%, 10 \%$ or $15 \%)$ applied to the price of this immunotherapy would have on the case base result. Against a maximum discount of 15\%, the savings associated with the SP142 assay in the base case would be reduced by $14.6 \%(€-4,856,984)$.

The BIM assumed that all patients actually eligible for the PD-L1 assay undergo a tissue biopsy. This may not match the actual clinical practice, as the PD-L1 assay is not always performed on a tissue biopsy sample, since this is sometimes not available or biopsy procedure is not clinically feasible, in which case the primary tumor tissue sample is used, if available. If the assumption made in the BIM overestimated the tissue biopsy cost, it is to be noted that this item does not represent an incremental cost for the two diagnostic alternatives, since, in both cases, all patients eligible for the PD-L1 assay undergo tissue biopsy.

The BIM evaluated the cost of the tissue biopsy considering the tariff reimbursed by the Tuscany Region for the outpatient service "histopathological examination of the breast - excision of neoformation" (code 91.42.B). In clinical practice, the tissue biopsy could also be executed for other sites (i.e., lymph nodes, lung); in this case the cost reimbursed by iNHS could be different from that used in the base case. Since the biopsy site stratification of patients is not available, it has been assumed that all patients eligible for the PD-L1 assay undergo the breast histopathological examination. To justify this assumption, we reported that any overestimation or underestimation of the related cost would be homogeneous for both the diagnostic alternatives (SP142 or 22C3).

The IMpassion130 data did not allow to distinguish between neutropenia and febrile neutropenia. Based on the literature, the estimated cost for grade $\geq 3$ neutropenia was $€ 2,390.11$. Since a grade $\geq 3$ non-febrile neutropenia may not involve any treatment or hospitalization, the sensitivity analysis also considered an alternative scenario, where no cost was associated with the presence of grade $\geq 3$ neutropenia. The result of this alternative scenario would determine a slight reduction in the savings associated with the use of the SP142 assay compared with the base case.

The present analysis has only evaluated the consequences deriving from the choice of one of the two different diagnostic strategies on total cost and median cost per PFS month, without considering the consequences that this choice could determine on the patient's overall survival.

It would also be good practice to discuss the results of a study by comparing them with those already published. Unfortunately, at the time of drafting this manuscript, no similar pharmacoeconomic evaluations are available in the literature to be used for this purpose.

\section{CONCLUSIONS}

These results show how the choice of a correct diagnostic strategy is important to optimize the administration of oncological therapies within the treatment of mTNBC patients. The use 
of the SP142 assay proved to be cost-effective compared to the 22C3 assay; the SP142 assay can support the choice of the most appropriate cancer drug, maximizing the effectiveness and minimizing the waste of healthcare resources.

\section{Funding}

This research was funded by an educational grant from Roche Diagnostic S.p.A. and Roche S.p.A. Italian affiliates of the Roche Group

\section{Conflict of interest}

CS declares consulting fees and honoraria from Roche outside the submitted work. RR and PR have nothing to disclose. MG declares consulting fees from Novartis, Pfizer, Roche, and Seagen; honoraria from Astrazeneca, Daiichi Sankyo, Lilly, Novartis, Pfizer, and Roche; support for attending meeting and/or travel from Lilly, Pfizer, Roche, and Daiichi Sankyo outside the submitted work.

\section{REFERENCES}

1. Vagia E, Mahalingam D, Cristofanilli M. The Landscape of Targeted Therapies in TNBC. Cancers (Basel). 2020;12:916; https://doi.org/10.3390/cancers12040916

2. Cyprian FS, Akhtar S, Gatalica Z, et al. Targeted immunotherapy with a checkpoint inhibitor in combination with chemotherapy: A new clinical paradigm in the treatment of triple-negative breast cancer. Bosn J Basic Med Sci 2019;19:227-33; https://doi.org/10.17305/bjbms.2019.4204

3. Schmid P, Rugo HS, Adams S, et al. Atezolizumab plus nab-paclitaxel as first-line treatment for unresectable, locally advanced or metastatic triple-negative breast cancer (IMpassion130): updated efficacy results from a randomised, double-blind, placebo-controlled, phase 3 trial. Lancet Oncol 2020;21:44-59; https://doi.org/10.1016/ S1470-2045(19)30689-8

4. Lehmann BD, Pietenpol JA, Tan AR. Triple-negative breast cancer: molecular subtypes and new targets for therapy. Am Soc Clin Oncol Educ Book 2015:e31-9; https://doi.org/10.14694/EdBook_AM.2015.35.e31

5. André F, Zielinski CC. Optimal strategies for the treatment of metastatic triple-negative breast cancer with currently approved agents. Ann Oncol 2012;23 Suppl 6:vi46-51; https://doi.org/10.1093/annonc/mds195

6. Vennapusa B, Baker B, Kowanetz M, et al. Development of a PD-L1 Complementary Diagnostic Immunohistochemistry Assay (SP142) for Atezolizumab. Appl Immunohistochem Mol Morphol 2019;27:92-100; https://doi. org/10.1097/PAI.0000000000000594

7. Tecentriq - Riassunto delle caratteristiche del prodotto. Available at: https://www.ema.europa.eu/en/documents/ product-information/tecentriq-epar-product-information_it.pdf (last accessed March 2020)

8. AIFA. Determina 14 luglio 2020 n. DG 757/2020. Regime di rimborsabilita' e prezzo, a seguito di nuove indicazioni terapeutiche, del medicinale per uso umano «Tecentriq» GU Serie Generale n.188 del 28-07-2020. Available at https:// www.gazzettaufficiale.it/atto/serie generale/caricaDettaglioAtto/originario?atto.dataPubblicazioneGazzetta=202007-28\&atto.codiceRedazionale=20A03900 (last accessed March 2020)

9. Package insert. Ventana PD-L1 (SP142) Assay REF 741-4860, 1018624IT Rev A. 2019-06-14 www.ventana.com

10. Rugo HS, Loi S, Adams S, et al. Performance of PD-L1 immunohistochemistry (IHC) assays in unresectable locally advanced or metastatic triple-negative breast cancer (mTNBC): Post-hoc analysis of IMpassion130. Ann Oncol 2019;30 (suppl_5):v851-v934; https://doi.org/10.1093/annonc/mdz394.009

11. Rugo HS, Loi S, Adams S, et al. Analytical Harmonization of PD-L1 Immunohistochemistry Assays in Advanced Triple-Negative Breast Cancer: A Retrospective Sub-Study of IMpassion130. Cancer Res 2020; 80(4 Supplement): PD1-07; https://doi.org/10.1158/1538-7445.SABCS19-PD1-07

12. Mauskopf JA, Sullivan SD, Annemans L, et al. Principles of good practice for budget impact analysis: report of the ISPOR Task Force on Good Research Practices-budget impact analysis. Value Health 2007;10:336-47; https:// doi.org/10.1111/j.1524-4733.2007.00187.x

13. Sullivan SD, Mauskopf JA, Augustovski F, et al. Budget impact analysis principles of good practice: report of the ISPOR 2012 Budget Impact Analysis Good Practice II Task Force. Value Health 2014;17:5-14; https://doi. org/10.1016/j.jval.2013.08.2291

14. Demo ISTAT 2020. Available at: http://www.demo.istat.it/pop2020/index.html (Last accessed March 2020) 
15. I numeri del Cancro in Italia 2020. Available at: https://www.aiom.it/wp-content/uploads/2020/10/2020_Numeri_Cancro-operatori_web.pdf(Last accessed March 2020)

16. ) I numeri del cancro in Italia 2019 (a cura di Stefania Gori). Available at: https:/www.aiom.it/wp-content/uploads/2019/09/2019_Numeri_Cancro-operatori-web.pdf (Last accessed March 2020)

17. AIOM. Linee Guida Neoplasie della Mammella. Edizione 2019. Available at: https://www.aiom.it/linee-guidaaiom-neoplasie-della-mammella-2019/ (Last accessed March 2020)

18. Gamucci T, Pizzuti L, Sperduti I, et al. Neoadjuvant chemotherapy in triple-negative breast cancer: A multicentric retrospective observational study in real-life setting. J Cell Physiol 2018;233:2313-23; https://doi.org/10.1002/ jcp.26103

19. Roche Diagnostic and Roche S.p.A. Data on file. IQVIA Ricerca di Mercato 2019/2020

20. Nomenclatore tariffario regionale delle prestazioni specialistiche ambulatoriali Regione toscana. Istopatologico mammella - escissione di neoformazione (Cod. 91.42.B). Available at: https://www.regione.toscana.it/ documents/10180/23313/nomenclatore+prestazioni+specialistica+ambulatoriale+.pdf/0386a27c-b47a-43d4-962315f20dbfa45d (Last accessed March 2020)

21. Tariffario delle prestazioni specialistiche della Regione Piemonte. Pannello di fattori prognostici e predittivi di risposta alla terapia per patologia tumorale maligna della mammella (Cod. 91.47.6). Available at: https://www. regione.piemonte.it/web/sites/default/files/media/documenti/2018-10/tariffario_prestazioni_specialistiche_rp.pdf (Last accessed March 2020)

22. Tariffario nazionale. Visita generale (cod. 89.7) Available at: https://www.trovanorme.salute.gov.it/norme/renderPdf. spring?seriegu=SG\&datagu=28/01/2013\&redaz=13A00528\&artp=3\&art=1\&subart=1\&subart $1=10 \&$ vers $=1 \& \mathrm{pr}$ og=001 (Last accessed March 2020)

23. Regione Lombardia. Deliberazione $n^{\circ} \mathrm{IX} / 2946$ seduta del 25/01/2012. Precisazioni in ordine alla DRG n. IX/2633 del 06 dicembre 2011 "Determinazioni in ordine alla gestione del servizio socio sanitario regionale per l'esercizio 2012. Available at https://www.assolombarda.it/fs/20122115055_141.pdf (Last accessed March 2020)

24. Lazzaro C, Bordonaro R, Cognetti F et al. An Italian cost-effectiveness analysis of paclitaxel albumin (nab-paclitaxel) versus conventional paclitaxel for metastatic breast cancer patients: the COSTANza study. ClinicoEconomics and Outcomes Research 2013;5:125-135; https://doi.org/10.2147/CEOR.S41850

25. Lazzaro C, Mazzanti NA, Parazzini F. Severe haematological toxicities in relapsed ovarian cancer treated with olaparib or niraparib: an Italian cost-minimization analysis. Global \& Regional Health Technology Assessment 2019;6:1-17; https://doi.org/10.1177/2284240319843941

26. Tariffario nazionale DRG. Tariffe delle prestazioni di assistenza ospedaliera per acuti, per tipo di ricovero (euro). https://www.trovanorme.salute.gov.it/norme/renderPdf.spring?seriegu=SG\&datagu=28/01/2013\&redaz=13A0052 8\&artp=1\&art=1\&subart=1\&subart 1=10\&vers=1\&prog=001 (Last access December 2020)

27. AIES, Associazione Italiana di Economia Sanitaria. Proposte di linee guida per la valutazione economica degli interventi sanitari. Pharmacoeconomics Italian Research Articles 2009;11:89-93; https://doi.org/10.1007/BF03320660 